\title{
Augmentation of systemic blood pressure during spinal cord ischemia to prevent postoperative paraplegia after aortic surgery in a rabbit model
}

So Izumi, MD, Kenji Okada, MD, Tomomi Hasegawa, MD, Atsushi Omura, MD, Hiroshi Munakata, MD, Masamichi Matsumori, MD, and Yutaka Okita, MD

Objective: Paraplegia from spinal cord ischemia remains an unresolved complication in thoracoabdominal aortic surgery, with high morbidity and mortality. This study investigated postoperative effects of systemic blood pressure augmentation during ischemia.

Methods: Spinal cord ischemia was induced in rabbits by infrarenal aortic occlusion for 15 minutes with infused phenylephrine (high blood pressure group, $\mathrm{n}=8$ ) or nitroprusside (low blood pressure group, $\mathrm{n}=8$ ) or without vasoactive agent (control, $\mathrm{n}=8$ ). Spinal cord blood flow, transcranial motor evoked potentials, neurologic outcome, and motor neuron cell damage (apoptosis, necrosis, superoxide generation, myeloperoxidase activity) were evaluated.

Results: Mean arterial pressures during ischemia were controlled at $121.9 \pm 2.8,50.8 \pm 4.3$, and $82.3 \pm 10.7 \mathrm{~mm}$ $\mathrm{Hg}$ in high blood pressure, low blood pressure, and control groups, respectively. In high blood pressure group, high spinal cord blood flow $(P<.01)$, fast recovery of transcranial motor evoked potentials $(P<.01)$, and high neurologic score $(P<.05)$ were observed after ischemia relative to low blood pressure and control groups. At 48 hours after ischemia, there were significantly more viable neurons, fewer terminal deoxynucleotidyl transferase-mediated deoxyuridine triphosphate nick-end labeling-positive neurons, and less $\alpha$-fodrin expression in high blood pressure group than low blood pressure and control groups. Superoxide generation and myeloperoxidase activity at 3 hours after ischemia were suppressed in high blood pressure group relative to low blood pressure group.

Conclusions: Augmentation of systemic blood pressure during spinal cord ischemia can reduce ischemic insult and postoperative neurologic adverse events. (J Thorac Cardiovasc Surg 2010;139:1261-8)

Spinal cord ischemia (SCI) is a major devastating and unpredictable complication in thoracoabdominal aortic surgery. Although SCI is not directly associated with high mortality, it may spoil the quality of life in patients and indirectly influence mortality. Many strategies have been devised to protect the spinal cord, including mild or deep hypothermia, distal aortic perfusion, segmental aortic clamping, reconstruction of intercostal or lumbar arteries, cerebrospinal fluid drainage, monitoring of motor evoked potentials, and pharmacologic agents. The reported incidence of paraplegia, however, still ranges from $2 \%$ to $11 \%{ }^{1-3}$ The mechanism for the development of paraplegia related to SCI is multifactorial, and this complication still cannot be prevented completely.

\footnotetext{
From the Department of Surgery, Division of Cardiovascular Surgery, Kobe University Graduate School of Medicine, Kobe, Japan.

Disclosures: None.

Received for publication May 12, 2009; revisions received July 15, 2009; accepted for publication Aug 9, 2009; available ahead of print Nov 12, 2009.

Address for reprints: Kenji Okada, MD, Department of Surgery, Division of Cardiovascular Surgery, Kobe University Graduate School of Medicine, 7-5-2, Kusunoki-Cho, Chuo-Ku, Kobe, Hyogo, 650-0017, Japan (E-mail: yutamo@aol.com). 0022-5223/\$36.00

Copyright (c) 2010 by The American Association for Thoracic Surgery doi:10.1016/j.jtcvs.2009.08.038
}

Some clinical and experimental reports have suggested that appropriate control of blood pressure (BP) could prevent SCI-related paraplegia after surgery. ${ }^{4-7}$ In this study, we hypothesized that intraoperative augmentation of systemic BP (SBP) during SCI could protect ischemic spinal cord injury. We investigated the impact of SBP augmentation on the spinal cord by means of neurophysiologic and histopathologic evaluations.

\section{MATERIALS AND METHODS \\ Animals}

Japanese white rabbits weighing 2.6 to $4.1 \mathrm{~kg}$ were obtained from Kitayama (Kitayama Labs Co Ltd, Nagano, Japan). The handling of laboratory animals and their use in experiments conformed to the "Guidelines for Animal Experiment at Kobe University Graduate School of Medicine',; in addition, all animals received humane care and treatment in accordance with the "Guide for the Care and Use of Laboratory Animals"' (www.nap.edu/ catalog/5140.html).

\section{Surgical Procedure}

Experiments were performed with a rabbit SCI model, which we have previously described. ${ }^{8}$ Briefly, rabbits anesthetized with intramuscular ketamine (Ketalar intramuscular, $50 \mathrm{mg} / \mathrm{kg}$; Daiichisankyo Co Ltd, Tokyo, Japan) and intravenous propofol (1\% Diprivan Injection, $10 \mathrm{mg} / \mathrm{kg}$; AstraZeneca, Boston, Mass) had a 5F balloon-tipped catheter (Swan-Ganz thermodilution catheter, 93-132-5F; Baxter Health Corporation, Santa Ana, 


\section{Abbreviations and Acronyms \\ $\mathrm{BP}=$ blood pressure \\ HBP $=$ high blood pressure \\ LBP $=$ low blood pressure \\ LLI = lower limb ischemia \\ MAP = mean arterial pressure \\ MTS = modified Tarlov scale \\ SBP = systemic blood pressure \\ $\mathrm{SCBF}=$ spinal cord blood flow \\ $\mathrm{SCI}=$ spinal cord ischemia \\ SSEP = somatosensory evoked potential \\ tc-MEP $=$ transcranial motor evoked potential \\ TUNEL $=$ terminal deoxynucleotidyl transferase- mediated deoxyuridine triphosphate nick-end labeling}

Calif) inserted into the abdominal aorta through the right superficial femoral artery. To establish the SCI model, the balloon of the catheter was inflated 0.5 to $1.5 \mathrm{~cm}$ distal to the left renal artery with an indicated SBP for $15 \mathrm{~min}-$ utes and then deflated with the SBP returned to normal level (approximately $80 \mathrm{~mm} \mathrm{Hg}$ ). For a preliminary study, the balloon was inflated at terminal aorta with an indicated SBP for 15 minutes to establish a lower-limb ischemia (LLI) model.

\section{Experimental Groups}

According to the SBP level during SCI, rabbits were randomly divided into 3 groups as follows. In the high $\mathrm{BP}(\mathrm{HBP})$ group $(\mathrm{n}=8)$, the mean arterial pressure (MAP) was maintained at $120 \mathrm{~mm} \mathrm{Hg}$ by an intravenous phenylephrine (Neo-Synesin Kowa Injection; Kowa Co Ltd, Tokyo, Japan). In the low BP (LBP) group $(\mathrm{n}=8)$, MAP was maintained at $50 \mathrm{~mm} \mathrm{Hg}$ by an intravenous nitroprusside (Nitopro continuous intravenous solution; Maruishi Pharmaceutical Co Ltd, Osaka, Japan). Finally, in the control group $(\mathrm{n}=8)$, MAP was approximately $80 \mathrm{~mm} \mathrm{Hg}$ without any additional medication. After the balloon deflation, we stopped using the vasoactive agents immediately, and the SBP was maintained naturally.

\section{Neurologic Assessment}

Serial assessments of motor function in the hind limbs of all animals were performed at 3, 24, and 48 hours after SCI according to a modified Tarlov scale (MTS) as described previously ${ }^{8}: 0$ for no movement, 1 for slight movement, 2 for sitting with assistance, 3 for sitting alone, 4 for weak hop, and 5 for normal hop).

\section{Measurement of Spinal Cord Blood Flow}

Spinal cord blood flow (SCBF) was measured as described previously ${ }^{9}$ with modifications. A laser probe (LP-N; Unique Medical Co Ltd, Tokyo, Japan) was used, and SCBF was continuously monitored until 30 minutes after the SCI by laser Doppler flowmetry (TBF-LC1; Unique Medical). After the SCBF baseline was recorded at $80 \mathrm{~mm} \mathrm{Hg}$, SCBFs at the indicated SBPs were measured in each group. The experimental SCBF was expressed as a percentage of the SCBF baseline.

\section{Measurement of Transcranial Motor Evoked Potentials}

Transcranial motor evoked potentials (tc-MEPs) were measured and analyzed with a modification of the method in our previous report. ${ }^{8}$ Tc-MEPs were evoked with a multiple transcranial electrical stimulator (NS-101 cor- tical stimulator; Unique Medical). Data acquisition, processing, analysis, and storage were performed with a personal computer system (UAS108S; Unique Medical). In this study, the tc-MEPs were measured every minute during the operation. The baseline for tc-MEPs was defined as an average of 3 consecutive amplitudes recorded before aortic occlusion, and the reappearance of tc-MEPs was defined as absence of flat waves in 3 consecutive responses. Recovery ratio of tc-MEPs amplitude was calculated as the amplitude of anterior tibial muscles divided by the baseline of anterior tibial muscles multiplied by the baseline of anterior radial muscles divided by the amplitude of anterior radial muscles and expressed as a percentage. ${ }^{10}$

\section{Evaluation of Pathologic Outcome}

Rabbits were killed by deep sodium pentobarbital anesthesia $(100 \mathrm{mg} / \mathrm{kg}$, intravenously) after 48 hours of reperfusion. The spinal cord between L3 and $\mathrm{L} 4$ was removed and placed in $4 \%$ paraformaldehyde $/ 0.1 \mathrm{~mol} / \mathrm{L}$ phosphatebuffered saline solution at $4{ }^{\circ} \mathrm{C}$ for 1 week. Sections were cut transversely at the L3 and L4 levels and embedded in paraffin. The sections were stained with hematoxylin and eosin for histopathologic observation of motor neurons, according to our previous report ${ }^{8}$ : viable neurons were indicated by basophilic stripling (containing Nissl substance), whereas nonviable neurons were indicated by pyknotic nuclei, eosinophilic cytoplasm, or absent nuclear hematoxylin staining. The numbers of viable neurons in unilateral Rexed laminae VII, VIII, and IX were counted and expressed as an average.

To detect DNA fragmentation in cell nuclei, the sections were also processed according to the terminal deoxynucleotidyl transferase-mediated deoxyuridine triphosphate nick-end labeling (TUNEL) method. The number of neurons with nuclei clearly stained by the TUNEL method was counted in the same way as the number of viable neurons.

\section{Western Blot Analysis}

Frozen spinal cord samples were homogenized, and the protein concentrations were evaluated with a dye-binding assay with the Bio-Rad reagent (Bio-Rad Laboratories, Hercules, Calif). Equal amounts of protein $(10 \mu \mathrm{g}$ protein per lane) were electrophoresed on a $10 \%$ sodium dodecyl sulfate polyacrylamide gel and then transferred onto nitrocellulose membrane. The membrane was incubated with anti-mouse $\alpha$-fodrin antibody (Millipore Corp, Billerica, Mass) for 1 hour at room temperature and then incubated with goat anti-mouse immunoglobulin antibody for 30 minutes. Enhanced chemiluminescence analysis was performed according to manufacturer instructions (GE Healthcare UK Limited, Buckinghamshire, UK). Blots were subsequently probed for $\beta$-actin (Bio Vision Research Products, Mountain View, Calif) as an internal control for equivalent protein loading. The signals were quantified with an image analyzer (LAS-3000; FUJIFILM Corp, Tokyo, Japan). Optical density of each band was measured on the same membrane.

\section{Superoxide Detection}

Superoxide generation was evaluated on tissue cryosections of the spinal cord between L3 and L4 at 3 hours after SCI. The sections were quickly removed, embedded in OCT compound (Sakura Finetech, Torrance, Calif), and stored at $-80{ }^{\circ} \mathrm{C}$ until use. Dihydroethidium (Invitrogen, Carlsbad, Calif) was used as an oxidative fluorescent dye.

\section{Myeloperoxidase Activity}

Tissue myeloperoxidase activity was determined by measuring the hydrogen peroxide-dependent oxidation of $o$-dianisidine as previously described, ${ }^{11}$ with modifications. In brief, frozen spinal cord samples were homogenized in hexadecyltrimethyl ammonium bromide (Sigma-Aldrich, St Louis, Mo) and phosphate-buffered saline solution at $\mathrm{pH}$ 6.0. The supernatants were reacted with $o$-dianisidine dihydrochloride (Sigma-Aldrich) and hydrogen peroxide. The change in absorbance was measured spectrophotometrically at $450 \mathrm{~nm}$. The activity was expressed as a percentage of that in the control group. 

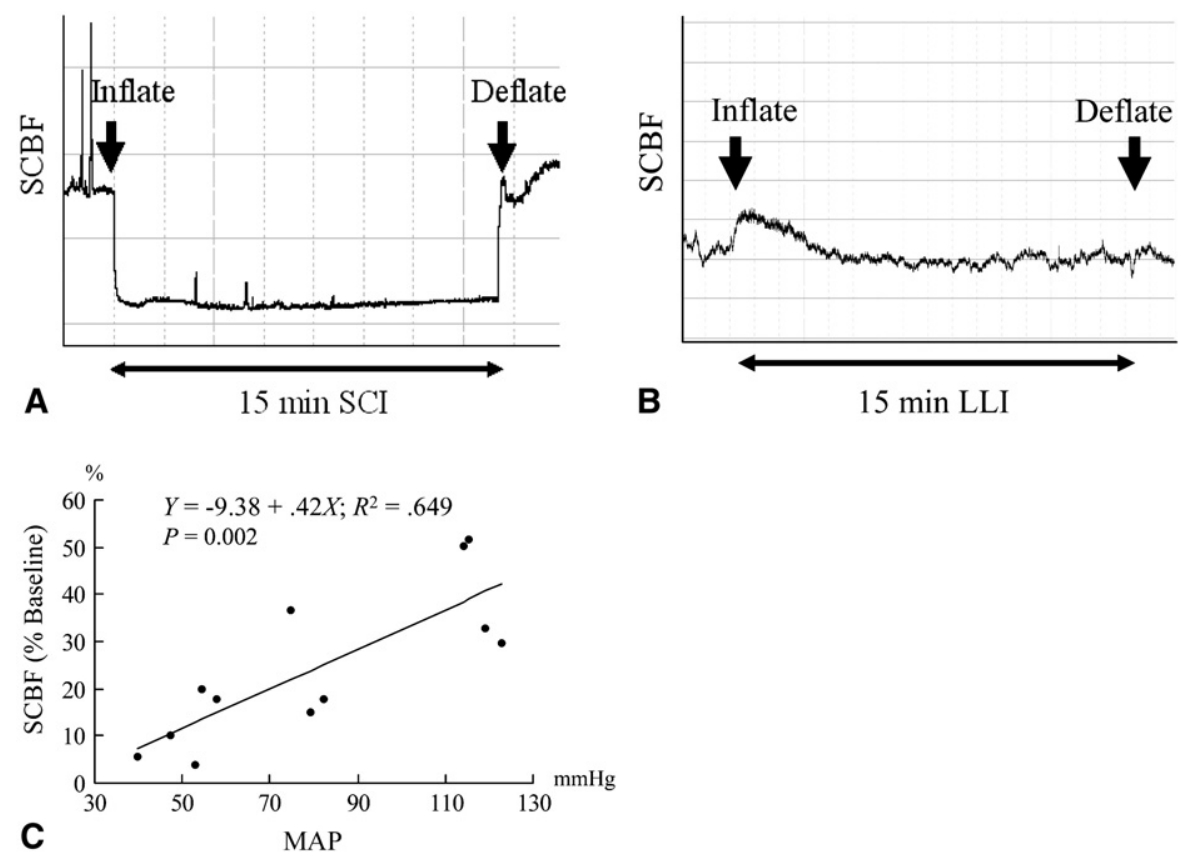

FIGURE 1. A, Spinal cord blood flow ( $S C B F$ ) recordings in spinal cord ischemia (SCI) model. B, Spinal cord blood flow recordings in lower limb ischemia (LLI) model. C, Relationship between mean arterial pressure $(M A P)$ and spinal cord blood flow during aortic occlusion in spinal cord ischemia model. Raw data shown as points.

\section{Statistical Analysis}

Data were processed with Stat View J-5.0 software (SAS Institute, Cary, $\mathrm{NC})$. All values are expressed as mean $\pm \mathrm{SD}$. Comparisons among groups were performed with Kruskal-Wallis test, Scheffé multiple comparison test, or repeated measures analysis of variance as appropriate.

\section{RESULTS}

\section{Rabbit SCI model}

To confirm our SCI model, experiments with a LLI model were performed in a preliminary study. The SCBF in the LLI model did not decrease during 15 minutes of the aortic occlusion, whereas that in the SCI model decreased dramatically and stayed low during the 15 minutes of aortic occlusion (Figure 1, $A$ and $B$ ). No animal in the LLI group had neurologic and histologic damage at 48 hours after reperfusion. On the basis of this result, we performed the following experiments with the rabbit SCI model.

\section{Intraoperative Physiologic Status}

In the SCI model, all animals survived for 48 hours after the balloon deflation. The intraoperative data are shown in Table 1. There were no statistical differences in intraoperative hemoglobin, arterial $\mathrm{PaO}_{2}$, and $\mathrm{pH}$ among the 3 groups. The MAP in the control group was $82.3 \pm 10.7 \mathrm{~mm} \mathrm{Hg}$. The average MAP during SCI was significantly different among the groups according to their definition $(P<.001)$. During SCI, the SCBF in HBP group was significantly higher than that in LBP or control group $(P<.001, P=.009$, respectively), whereas no significant difference was found be- tween the LBP and control groups. A clear correlation between MAP and SCBF was observed during SCI (relation coefficient $=0.81, P=.002$; Figure $1, C$ ).

\section{Intraoperative and Postoperative Neurologic Evaluation}

The tc-MEPs disappeared immediately after aortic occlusion and reappeared after balloon deflation. The recovery of tc-MEPs is shown in Figure $2(A$ and $B$ ). The tc-MEPs in the HBP group reappeared only $3.8 \pm 5.6$ minutes after SCI, whereas the reappearance times for the LBP and control groups were $22.2 \pm 5.2$ minutes and $21.0 \pm 6.7$ minutes, respectively. The reappearance time of tc-MEPs in the HBP group was significantly faster than that in either the LBP group or the control group $(P=.005, P=.008$, respectively). The recovery ratio of tc-MEP amplitude through 30 minutes after SCI was also significantly larger in the

TABLE 1. Intraoperative physiologic status by group

\begin{tabular}{lccc}
\hline & $\begin{array}{c}\text { High blood } \\
\text { pressure }\end{array}$ & $\begin{array}{c}\text { Low blood } \\
\text { pressure }\end{array}$ & Control \\
\hline $\mathrm{pH}$ & $7.34 \pm 0.04$ & $7.37 \pm 0.01$ & $7.38 \pm 0.05$ \\
Arterial $\mathrm{PaO}_{2}(\mathrm{~mm} \mathrm{Hg})$ & $94.0 \pm 16.0$ & $90.0 \pm 15.8$ & $99.7 \pm 17.0$ \\
Hemoglobin $(\mathrm{g} / \mathrm{dL})$ & $12.6 \pm 0.3$ & $12.3 \pm 1.1$ & $11.5 \pm 0.9$ \\
Mean arterial pressure $(\mathrm{mm} \mathrm{Hg})$ & $121.9 \pm 2.8^{*}$ & $50.8 \pm 4.3^{*}$ & $82.3 \pm 10.7$ \\
Spinal cord blood flow $(\%)$ & $45.2 \pm 7.2 \dagger \dagger$ & $11.7 \pm 6.7$ & $23.8 \pm 8.4$ \\
\hline $\begin{array}{l}\text { All values are mean } \pm \mathrm{SD} . \\
\text { versus low blood pressure. }\end{array}$ &
\end{tabular}




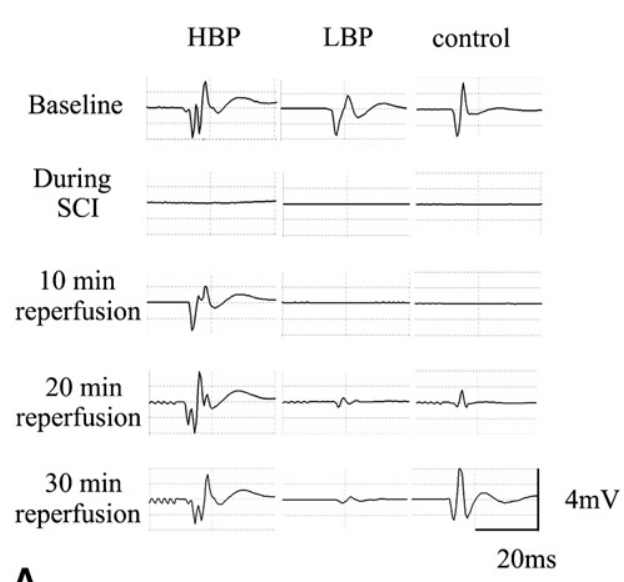

A

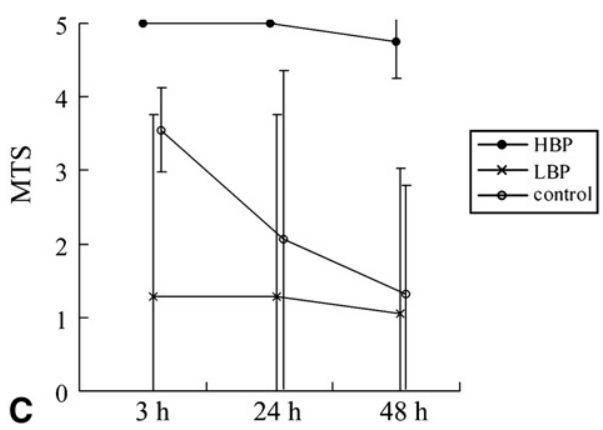

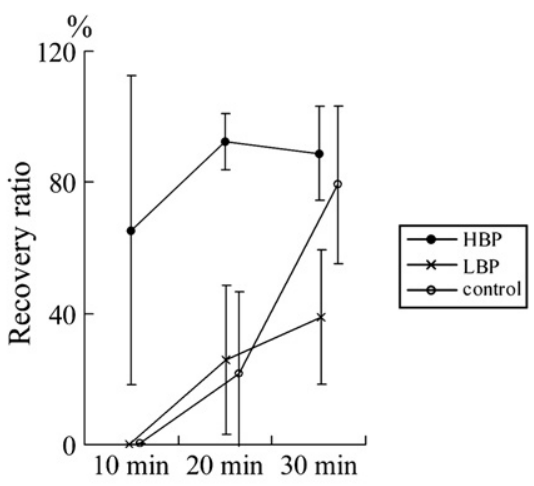

B

FIGURE 2. Intraoperative and postoperative neurologic assessments. A, Representative transcranial motor evoked potential (tc-MEP) complex. B, Recovery ratios of transcranial motor evoked potential amplitude at 10,20, and 30 minutes after spinal cord ischemia (SCI). C, Neurologic scores with modified Tarlov scale $(M T S)$ at 3, 24, and 48 hours after spinal cord ischemia. $H B P$, High blood pressure; $L B P$, low blood pressure.

HBP group than in either the LBP group or the control group $(P=.004$ and $P=.01$ by analysis of variance, respectively). MTS scores for each group are shown in Figure $2(C)$. No rabbits in the HBP group were neurologically damaged at 3, 24, and 48 hours after the MTS, although 48 hours after the SCI in HBP group was significantly higher than that in LBP or control group $(P=.01, P=.01$, by analysis of variance, respectively). There were no significant differences in intraoperative and postoperative neurologic evaluations between the LBP and control groups.

\section{Histologic Assessment}

At 48 hours after SCI, the number of viable motor neurons in the HBP group was significantly more than those in the LBP and control groups $(23.3 \pm 5.5,8.5 \pm 5.0$, and $6.2 \pm$ 3.8 per unilateral section, respectively, HBP vs LBP $P<$ .001 , HBP vs control $P<.001$; Figure $3, A$ and $C$ ). On the other hand, there were no significant differences between LBP and control group. The numbers of TUNEL-positive neurons per unilateral section in HBP, LBP, and control groups at 48 hours after SCI were $0.8 \pm 0.9,4.0 \pm 4.4$, and $12.8 \pm 5.8$, respectively (Figure $3, B$ and $D$ ). The number of TUNEL-positive neurons in HBP group was significantly less than that in control group $(P=.01)$, whereas there were no significant difference between HBP and LBP groups. The number of TUNEL-positive neurons in the control group was significantly larger than that in the LBP group $(P=.04)$.

\section{Expression of $\alpha$-Fodrin Fragments}

To quantify neuronal damage after SCI, we evaluated the protein expression of $\alpha$-fodrin in the spinal cord at 48 hours after SCI by Western blot analysis (Figure 4). The 120-, 145and $150-\mathrm{kDa}$ fragments of $\alpha$-fodrin are generated in accordance with proteolysis of a membrane cytoskeletal protein by neuronal damage of brain or spine. ${ }^{12-14}$ The total $\alpha$-fodrin level in the HBP group was significantly lower than those in both the LBP and control groups $(P<.001, P<.001$, respectively). There was also a significant difference between the LBP and control groups $(P=.004)$.

\section{Oxidative Stress}

For better understanding of the protective effect of HBP during SCI, we evaluated the superoxide generation and myeloperoxidase activity in the spinal cord at 3 hours after SCI. The intensity of red oxidative fluorescence in the HBP group was apparently lower than in either the LBP or 
HBP
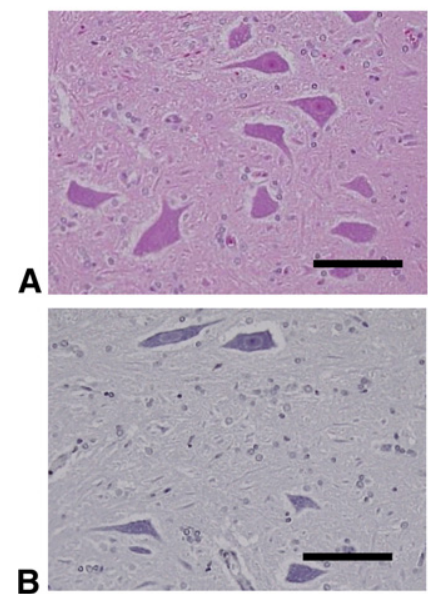

LBP
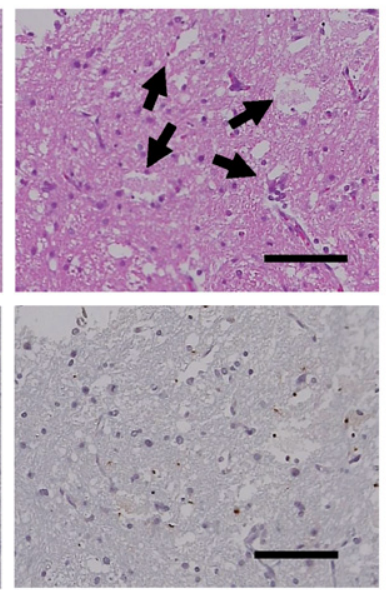

control
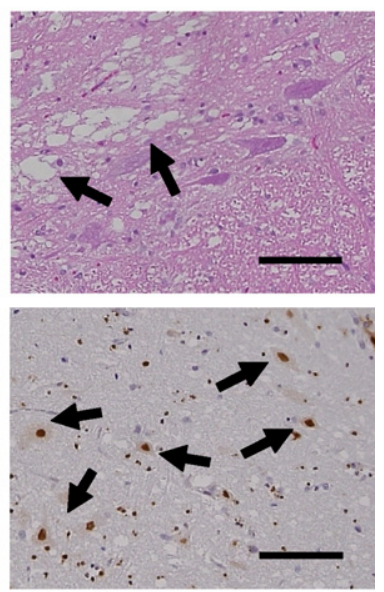
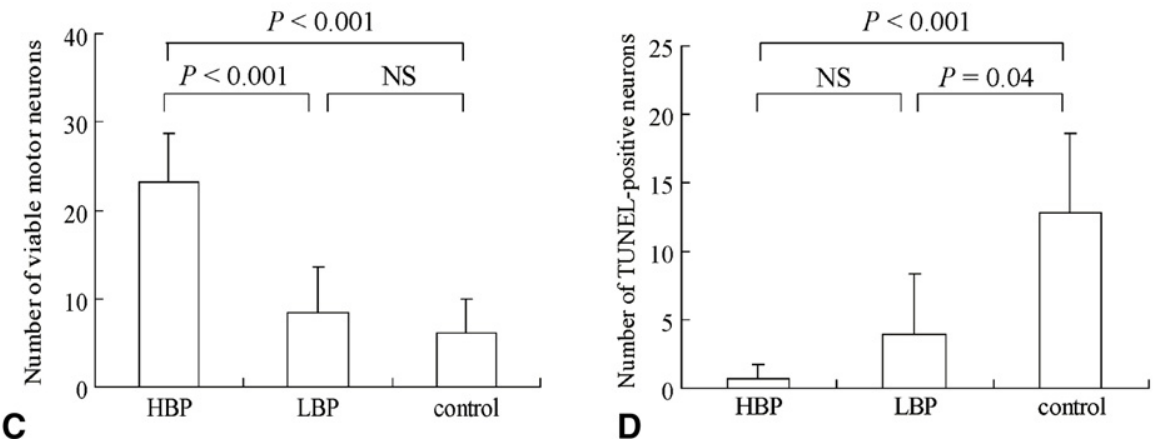

FIGURE 3. Photomicrographs of histologic sections in rabbit spinal cord at 48 hours after spinal cord ischemia. A, Hematoxylin and eosin staining of ventral gray matter. Spinal cords in low blood pressure $(L B P)$ and control groups show necrotic change (arrows). B, Terminal deoxynucleotidyl transferase-mediated deoxyuridine triphosphate nick-end labeling (TUNEL) staining of ventral gray matter. There were many positively staining neurons in control group (arrows). $\mathrm{C}$, Numbers of viable motor neurons in each group at 48hours after spinal cord ischemia. D, Numbers of terminal deoxynucleotidyl transferase-mediated deoxyuridine triphosphate nick-end labeling-positive neurons in each group at 48 hours after spinal cord ischemia. NS, Not significant. Scale bar represents $100 \mu \mathrm{m}$.

control group (Figure 5, A). Myeloperoxidase activities in the HBP and LBP groups were $86.4 \% \pm 34.2 \%$ and $181.4 \% \pm 68.6 \%$ of those in the control group, respectively. The myeloperoxidase activity in the LBP group was significantly higher than those in the HBP and control groups $(P=.007, P=.03$, respectively), whereas there was no significant difference between the HBP and control groups (Figure 5, $B$ ).

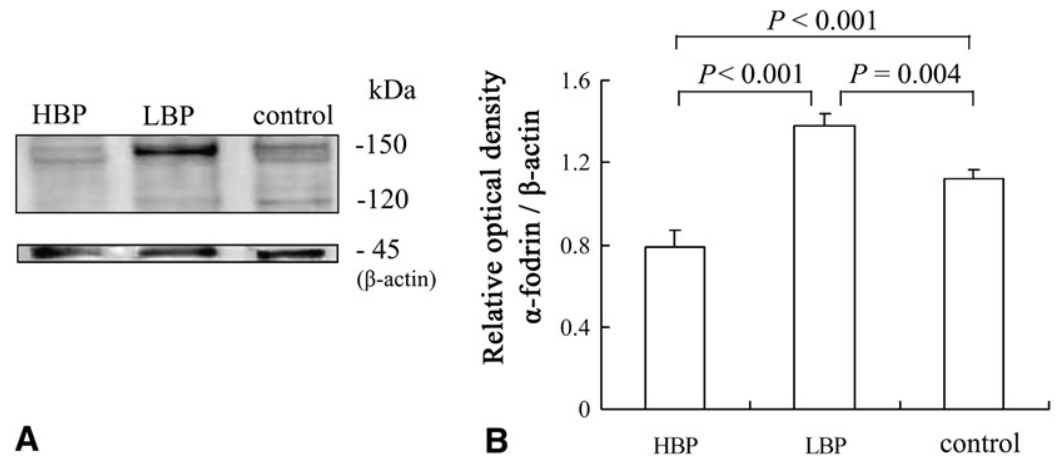

FIGURE 4. A, Western blot analysis of $\alpha$-fodrin fragments at 48 hours after spinal cord ischemia. B, Relative optical densities of total $\alpha$-fodrin fragments in each group at 48 hours after spinal cord ischemia. $H B P$, High blood pressure; $L B P$, low blood pressure. 

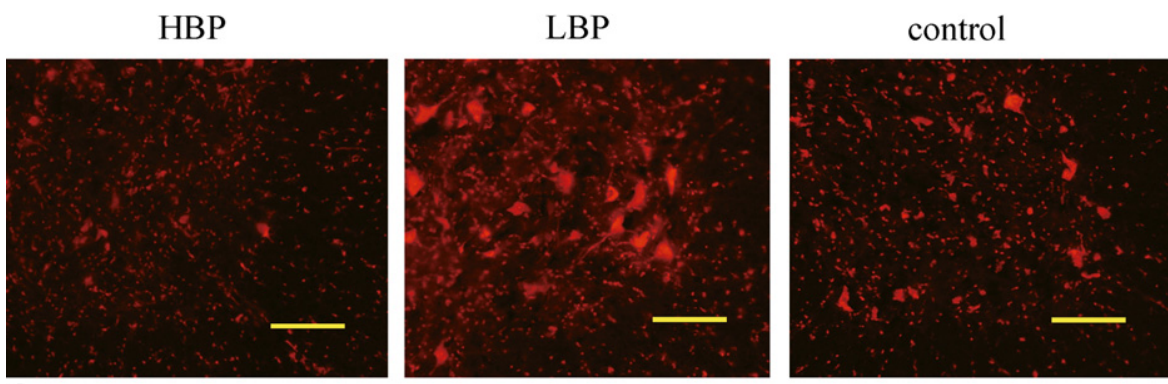

A

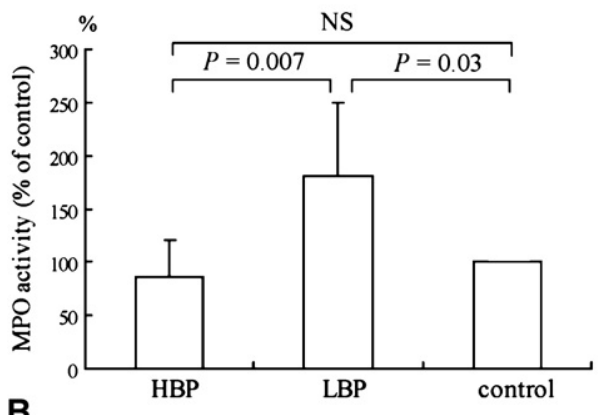

B

FIGURE 5. A, Fluorescence micrographs of histologic sections in rabbit spinal cord at 3 hours after spinal cord ischemia. Dihydroethidium staining (red fluorescence when oxidized to ethidium bromide by superoxide) of ventral horn. B, Myeloperoxidase (MPO) activities in each group at 3 hours after spinal cord ischemia. $H B P$, High blood pressure; $L B P$, low blood pressure; NS, not significant. Scale bar represents $200 \mu \mathrm{m}$.

\section{DISCUSSION}

The rabbit model involving infrarenal aortic clamping for 15 minutes is well established as a model of late paraplegia after SCI. ${ }^{13,15}$ The rabbit spinal cord is large even in the lumbar segment and extends up to sacral canal, so simple infrarenal aortic occlusion easily leads to paraplegia. ${ }^{16}$ Before starting this study, we preliminarily confirmed the appropriateness of our rabbit SCI model by comparing it with a rabbit LLI model.

Lu and colleagues ${ }^{5}$ and Taira and associates ${ }^{6}$ reported that in rat models aortic occlusion with hypovolemic hypotension caused more profound spinal cord hypoperfusion, resulting in severe ischemic injury of the spinal cord relative to normal arterial pressure. Although intraoperative hypotension as a result of blood loss is a common pathway to postoperative paraplegia, the decrease in perfusion pressure, hemoglobin, and circulatory volume associated with the blood loss are confounding factors in accurately evaluating the effect on the spinal cord of BP during aortic crossclamping. The effects on SCI of hypovolemia and anemia induced by exsanguination were not excluded in previous models. Recently, Toung and colleagues ${ }^{7}$ reported that hypertension during aortic clamping improved neurologic outcome in a rat model. Although postoperative neurologic and histologic assessments were performed in that study, they did not show any actual data of SCI demonstrated by SCBF and tc-MEPs in their rat experimental model. The utility of their rat model thus remains unclear.
In this study, SBP during SCI had a positive effect on SCBF in a rabbit model. Increased collateral flow possibly contributed to maintain sufficient SCBF during SCI in the HBP group. Although there are some collaterals from pial anastomoses through the posterior spinal artery to the lumbar cord, the caudal SCBF is mainly supplied from the segmental arteries in rabbits. ${ }^{16}$ On the other hand, there are many collaterals from the lumbar and internal iliac arteries through the anterior spinal artery to the caudal spinal cord in human beings. ${ }^{17,18}$ Although the effect of collateral supply in rabbits is believed to be smaller than in human beings, the increased collateral flow may have protected motor neurons and prevented paraplegia in this study.

Spinal cord integrity can be monitored with somatosensory evoked potentials (SSEPs), and myogenic motor evoked responses with tc-MEPs. Because SSEPs provide false-negative results and slow responses, tc-MEPs tend to be used as the SCI monitor during thoracoabdominal aortic aneurysm surgery. ${ }^{19,20} \mathrm{We}$ recently used tc-MEPs to analyze SCI experimentally and clinically. ${ }^{8,10}$ In this study, the time for tc-MEPs reappearance in the HBP group was faster, and the recovery ratio of tc-MEP amplitude through 30 minutes after reperfusion was larger than in the LBP and control groups. At 48 hours after SCI in this study, all rabbits in the HBP group did not demonstrate paraplegia, whereas most of the rabbits in the LBP and control groups demonstrated paraplegia. These findings suggest that slow recovery of tc-MEPs after SCI predicts poor neurologic outcome, as 
previous reports have suggested. ${ }^{8,10,21,22}$ Although half of the rabbits in the control group recovered to the baseline level at 30 minutes after reperfusion, they showed delayed paraplegia. The irreversible change in motor neurons thus may occur after the monitoring of tc-MEPs.

Histologic analysis in this study demonstrated that histologic damage was attenuated in HBP group, whereas significant damage was observed in both the LBP and control groups. There were also many TUNEL-positive neurons in the control group. In addition, the result of Western blot analysis suggested that neuronal death was most suppressed in the HBP group, whereas neuronal death was most facilitated in the LBP group among the 3 groups. The $120-\mathrm{kDa}$ fragment of $\alpha$-fodrin, which is a specific marker for apoptosis, ${ }^{14}$ appeared in the LBP and control groups. Sakurai and coworkers ${ }^{15}$ reported that apoptosis plays an important role in delayed paraplegia in the rabbit SCI model. In this study, the histologic and biochemical analyses suggest that delayed paraplegia may have been associated with apoptosis. On the other hand, rabbits in the LBP group both tended to show early paraplegia and had few TUNEL-positive neurons. Although we were unable to quantify the accurate number of necrotic neurons because of severe destruction and vacuolization of the gray matter, the spinal cord section in the LBP group tended to show characteristics of necrotic tissue. Further study is needed to elucidate a relationship between apoptosis or necrosis of motor neurons and paraplegia induced by SCI.

One of the factors involved in the development of ischemia-induced spinal cord injury is thought to be oxidative stress. Ege and colleagues ${ }^{23}$ reported that a free-radical scavenger attenuated oxidative stress, and neurologic outcomes were improved in their rabbit SCI models. In this study, superoxide generation and myeloperoxidase activity were facilitated in the LBP group at 3 hours after SCI. Hypoperfusion during SCI may facilitate oxidative stress and subsequent neuronal damage and thus may cause early paraplegia. Meanwhile, superoxide generation and myeloperoxidase activity were suppressed in the HBP group at 3 hours after SCI relative to the LBP group. Sufficient blood flow during SCI may suppress oxidative stress, which could contribute to spinal cord protection.

Although this experiment supports the hypothesis that perfusion pressure in the upper spinal arteries should be increased by the infrarenal aortic occlusion, it also proves that increasing perfusion pressure only by aortic occlusion without phenylephrine (control group) is insufficient to prevent postoperative paraplegia. Pharmacologic intervention to increase perfusion pressure during aortic occlusion (HBP group) significantly prevented postoperative paraplegia in this experiment. Clinically, this study indicates that the maintenance of collateral flow could be effective in spinal cord protection, corresponding with the concept of the distal aortic perfusion. Distal aortic perfusion with left heart by- pass or partial cardiopulmonary bypass has been clinically applied to maintain sufficient collateral flow to the spinal cord during aortic crossclamping, leading to decreased incidence of paraplegia. ${ }^{1,2} \mathrm{We}$ and others ${ }^{2,4}$ have reported proximal MAP to be clinically maintained between 60 and 100 $\mathrm{mm} \mathrm{Hg}$ and distal perfusion pressure kept above $70 \mathrm{~mm}$ $\mathrm{Hg}$ during aortic crossclamping.

One of the study limitations is that the normal BP in rabbits is different from that in human beings. The MAP ranged from 64.4 to $66.0 \mathrm{~mm} \mathrm{Hg}$ in a study on BP in conscious rabbits. ${ }^{24}$ Surgical procedure and aortic occlusion may influence BP, thus making the MAP in the control group relatively high in our study. Another limitation is that spinal cord vasculature in rabbits may be different from that in human beings. Another study limitation is that the SCBF may not have exactly reflected the blood flow of the anterior spinal artery because the SCBF was measured at the posterior side of the spinal cord. A final limitation is that this study could not simulate the situation in which the patient has coagulopathy and must be kept relatively hypotensive for a time to control the bleeding in clinical settings. To evaluate the effect of BP during aortic crossclamping on the spinal cord accurately, we eliminated the potential effects of hypovolemia, anemia, or reperfusion pressure in our study.

In conclusion, SBP augmentation during SCI in a rabbit model maintained sufficient SCBF so that ischemic insult to spinal cord and postoperative neurologic damage were reduced. This study suggests that it is important to maintain adequate $\mathrm{BP}$ for spinal cord protection during thoracoabdominal aortic surgery.

We thank Dr Yoshimasa Maniwa (Department of Surgery, Division of Thoracic Surgery, Kobe University Graduate School of Medicine) and Dr Yoshitake Hayashi (Division of Molecular Medicine \& Medical Genetics, International Center for Medical Research and Treatment, Kobe University Graduate School of Medicine) for their kind support with the Western blot analysis.

\section{References}

1. Safi HJ, Miller CC III, Huynh TT, Estrera AL, Porat EE, Winnerkvist AN, et al. Distal aortic perfusion and cerebrospinal fluid drainage for thoracoabdominal and descending thoracic aortic repair: ten years of organ protection. Ann Surg. 2003; 238:372-80.

2. Schepens M, Dossche K, Morshuis W, Heijmen R, van Dongen E, Ter Beek H, et al. Introduction of adjuncts and their influence on changing results in 402 consecutive thoracoabdominal aortic aneurysm repairs. Eur J Cardiothorac Surg. 2004;25:701-7.

3. Coselli JS, LeMaire SA, Miller CC 3rd, Schmittling ZC, Koksoy C, Pagan J, et al. Mortality and paraplegia after thoracoabdominal aortic aneurysm repair: a risk factor analysis. Ann Thorac Surg. 2000;69:409-14.

4. Kawanishi Y, Okada K, Matsumori M, Tanaka H, Yamashita T, Nakagiri K, et al. Influence of perioperative hemodynamics on spinal cord ischemia in thoracoabdominal aortic repair. Ann Thorac Surg. 2007;84:488-92.

5. Lu K, Liang CL, Chen HJ, Chen SD, Hsu HC, Liliang PC, et al. Injury severity and cell death mechanisms: effects of concomitant hypovolemic hypotension on spinal cord ischemia-reperfusion in rats. Exp Neurol. 2004;185:120-32.

6. Taira Y, Marsala M. Effect of proximal arterial perfusion pressure on function, spinal cord blood flow, and histopathologic changes after increasing intervals of aortic occlusion in the rat. Stroke. 1996;27:1850-8. 
7. Toung TJ, Chang Y, Williams M, Crain BJ, Traystman RJ, Bhardwaj A. Experimental spinal cord ischemia: model characterization and improved outcome with arterial hypertension. Crit Care Med. 2004;32(6):1346-51.

8. Murakami H, Tsukube T, Kawanishi Y, Okita Y. Transcranial myogenic motorevoked potentials after transient spinal cord ischemia predicts neurologic outcome in rabbits. J Vasc Surg. 2004;39:207-13.

9. Lindsberg PJ, O’Neill JT, Paakkari IA, Hallenbeck JM, Feuerstein G. Validation of laser-Doppler flowmetry in measurement of spinal cord blood flow. Am J Physiol. 1989;257(2 Pt 2):H674-80.

10. Kawanishi Y, Munakata H, Matsumori M, Tanaka H, Yamashita T, Nakagiri K, et al. Usefulness of transcranial motor evoked potentials during thoracoabdominal aortic surgery. Ann Thorac Surg. 2007;83:456-61.

11. Gao F, Chen J, Lopez BL, Christopher TA, Gu J, Lysko P, et al. Comparison of bisoprolol and carvedilol cardioprotection in a rabbit ischemia and reperfusion model. Eur J Pharmacol. 2000;406:109-16.

12. Fukuda S, Harada K, Kunimatsu M, Sakabe T, Yoshida K. Postischemic reperfusion induces alpha-fodrin proteolysis by m-calpain in the synaptosome and nucleus in rat brain. $J$ Neurochem. 1998;70:2526-32.

13. Kiyoshima T, Fukuda S, Matsumoto M, Iida Y, Oka S, Nakakimura K, et al. Lack of evidence for apoptosis as a cause of delayed onset paraplegia after spinal cord ischemia in rabbits. Anesth Analg. 2003;96:839-46.

14. Wang KK, Posmantur R, Nath R, McGinnis K, Whitton M, Talanian RV, et al. Simultaneous degradation of alphaII- and betaII-spectrin by caspase 3 (CPP32) in apoptotic cells. J Biol Chem. 1998;273:22490-7.

15. Sakurai M, Hayashi T, Abe K, Sadahiro M, Tabayashi K. Delayed and selective motor neuron death after transient spinal cord ischemia: a role of apoptosis? $J$ Thorac Cardiovasc Surg. 1998;115:1310-5.
16. DeGirolami U, Zivin JA. Neuropathology of experimental spinal cord ischemia in the rabbit. J Neuropathol Exp Neurol. 1982;41:129-49.

17. Lazorthes G, Gouaze A, Zadeh JO, Santini JJ, Lazorthes Y, Burdin P. Arterial vascularization of the spinal cord. Recent studies of the anastomotic substitution pathways. J Neurosurg. 1971;35:253-62.

18. Backes WH, Nijenhuis RJ, Mess WH, Wilmink FA, Schurink GW, Jacobs MJ. Magnetic resonance angiography of collateral blood supply to spinal cord in thoracic and thoracoabdominal aortic aneurysm patients. J Vasc Surg. 2008;48: 261-71.

19. Jacobs MJ, Meylaerts SA, de Haan P, de Mol BA, Kalkman CJ. Strategies to prevent neurologic deficit based on motor-evoked potentials in type I and II thoracoabdominal aortic aneurysm repair. J Vasc Surg. 1999;29:48-57.

20. Meylaerts SA, Jacobs MJ, van Iterson V, De Haan P, Kalkman CJ. Comparison of transcranial motor evoked potentials and somatosensory evoked potentials during thoracoabdominal aortic aneurysm repair. Ann Surg. 1999;230:742-9.

21. de Haan P, Kalkman CJ, de Mol BA, Ubags LH, Veldman DJ, Jacobs MJ. Efficacy of transcranial motor-evoked myogenic potentials to detect spinal cord ischemia during operations for thoracoabdominal aneurysms. J Thorac Cardiovasc. Surg. 1997;113:87-100.

22. van Dongen EP, Schepens MA, Morshuis WJ, ter Beek HT, Aarts LP, de Boer A, et al. Thoracic and thoracoabdominal aortic aneurysm repair: use of evoked potential monitoring in 118 patients. J Vasc Surg. 2001;34:1035-40.

23. Ege E, Ilhan A, Gurel A, Akyol O, Ozen S. Erdosteine ameliorates neurological outcome and oxidative stress due to ischemia/reperfusion injury in rabbit spinal cord. Eur J Vasc Endovasc Surg. 2004;28:379-86.

24. Brooks B, Muirhead EE. Routine aortic pressure measurements of the conscious rabbit. Arch Pathol. 1972;93:464-6. 